Ann. Génét. Sél. Anim., I979, 11 (4), 381-39o.

\title{
Étude de quelques variants colorés du porc Gréole de la Guadeloupe
}

\author{
J. J. LaUvergne, I. Canope * \\ Département de Génétique Animale, I.N.R.A., Centre National de Recherches Zootechniques, \\ 78350 Jouy-en-Josas \\ * Centre de Recherches Agronomiques Antilles-Guyane, 97170 Petit-Bourg, Guadeloupe
}

\section{Résumé}

Lors de croisements réalisés à l'élevage expérimental de porcs Créoles de la Guadeloupe au C.R.A.A.G. (Petit-Bourg, Guadeloupe), ont été mis en évidence des variants colorés qui, apparemment, correspondent à des allèles déjà connus chez le porc européen, aux deux loci indépendants $\mathrm{E}$ (Extension $d u$ noir) et I (Rouan).

$\mathrm{En} \mathrm{E}$ on aurait $E$ (Extension normale) dominant $E^{p}$ (ou $\left.e^{j}\right)$ noir et rouge (ou tigré); en $\mathrm{I}$ on aurait $I^{d}$ (gris) dominant $i$ (intense).

On ne note apparemment aucune ségrégation au locus $A$ ( $A$ gouti) dont la formule pour tous les animaux de l'expérience pourrait être $a a$ (noir recessif).

Certains sujet portaient des panachures blanches (ceinture ou socquettes, par ex.) mais, faute de relevés précis, l'étude génétique n'a pas pu en être faite.

La formule génétique colorée des porcs noirs serait $E E$ (ou $E E^{p}$ ) aa ii celle des porcs noir et rouge localement appelés "domino" et dont le dessin est variable quant à l'extension des taches noires et l'intensité du fond rouge serait $E^{p} E^{p}$ aa ii. Les porcs gris seraient quant à eux $E E$ (ou $E E^{p}$ ) $a a I^{d} i$ cependant que les animaux dits "chabin" (pelage blanc avec quelques taches cutannées noires) observés seraient $E^{p} E^{p} a a I^{d} i$. Toutefois, la pénétrance de l'hétérozygote $I^{d} i$ pour donner du chabin avec $E^{p} E^{p} a a$ ne semble pas totale ce qui fait qu'un certain nombre d'animaux $E^{p} E^{p}$ aa $I^{\tilde{d}_{i}}$ présentaient le phénotype domino.

\section{Introduction}

Le porc autochtone de la Guadeloupe appelé porc Créole ou porc Planche a une origine qui remonterait à des lâchers par les Espagnols dès le xve siècle (DUTERTRE, I667-7I; LABAT, I670).

Ses caractéristiques zootechniques ont été précisées par LE MEN'TEC (I978). Il s'agit d'un animal présentant toute une gamme de phénotypes colorés. 
Un élevage expérimental de porcs Créoles a été créé au Centre de Recherches Agronomiques Antilles-Guyane (C.R.A.A.G.), à Petit-Bourg, Basse-Terre, Guadeloupe. Ainsi ont pu être faites des observations sur la ségrégation des facteurs de coloration du pelage, qui sont analysés dans le présent article.

\section{Matériel et méthode}
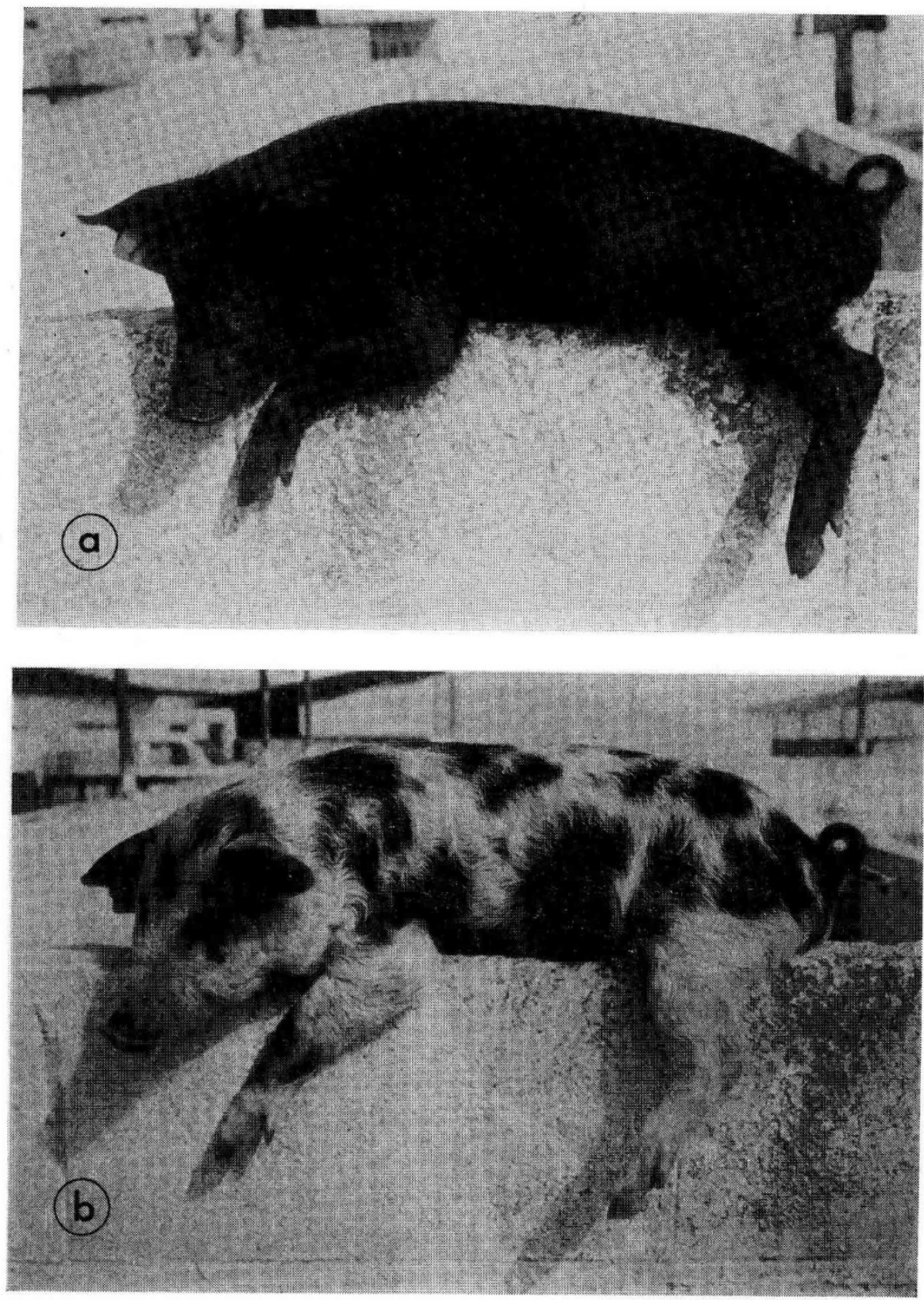

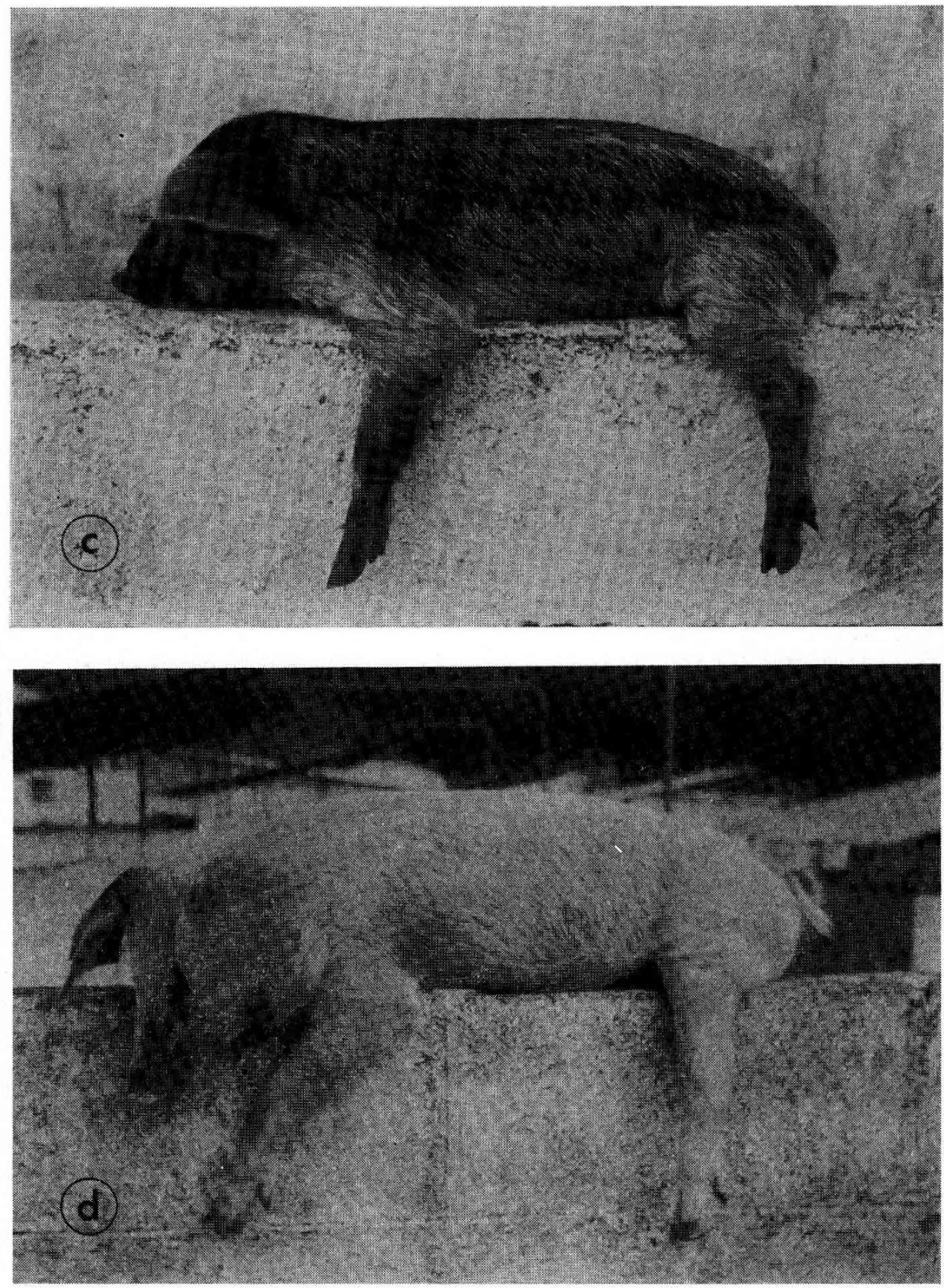

FIG. I. - Les phénotypes pour les couleurs pigmentaires.

a) Noir.

b) Domino (noir et rouge ou tigré). c) Gris.

d) Chabin (sorte de blanc sale).

Pigmentary colour phenotypes.
a) Black.
c) Grey (or roan).
b) Domino (black and red or tigering).
d) Chabin (kind of dirty white). 


\section{I. - Le matériel}

Au total 48 portées ont été observées entre I977 et I979. On a noté la couleur pigmentaire et les panachures. Toutefois l'enregistrement des panachures était succinct et les données peu nombreuses si bien que l'étude présente portera sur les patrons pigmentaires c'est-à-dire la répartition des deux types de mélanine phœeo et eumélanine dans le poil et le pelage, à l'exclusion des dessins blancs qui sont causés par des absences locales de pigment.

\section{2. - L'échelle des phénotypes pigmentaire}

On a distingué les 4 phénotypes pigmentaires suivants :

- Le noir (cf. fig. Ia).

- Le domino : cette appellation créole imagée désigne ici des animaux présentant des taches noires sur fond blanc. A première vue on pourrait être tenté de penser qu'il s'agit d'une panachure irrégulière mais, en fait, il s'agit bien d'une couleur pigmentaire car le fond lorsqu'il est blanc n'est en fait qu'un rouge dilué et, outre qu'il naît fréquemment des animaux tachés de noir sur fond rouge il est souvent possible de trouver sur un vrai domino des petites taches rouges. Dans le présent article nous avons étendu l'appellation domino aux phénotypes avec toutes les teintes possibles du fond rouge, du rouge franc au rouge très dilué quasiment blanc, avec toutes les extensions possibles des taches noires (cf. fig. $\mathrm{x} b$ ).

- Le gris : cette couleur est données par un mélange de poils noirs et blancs. On distingue parfois des stries horizontales plus foncées (cf. fig. Ic).

- Le chabin : le pelage est blanc et la peau est dépigmentée sur sa plus grande surface sauf quelques petites zones qui ont alors un aspect blanc sale (cf. fig. Id).

\section{3. - Échelle des panachures}

On note pour mémoire les dessins suivants :

- La ceinture plus ou moins étendue.

- Les socquettes blanches dont l'extension vers le haut des pattes est variable.

Ces dessins sont nets sur fond pigmentaire noir. Lorsqu'on est sur fond domino avec atténuation du rouge la détection du dessin socquettes blanches en particulier peut être délicate.

La variabilité des dessins blancs est assez grande et une étude sérieuse necessiierait le silhouettage systématique or celui-ci n'a pas été fait pour les données dont nous disposons d'où l'impossibilité déjà notée où nous sommes de les analyser d'une manière convenable.

\section{4. - Méthodes d'analyse des données}

On a considéré 1'ajustement des données d'observation aux modèles classiques de "monohybridisme " et de " dihybridisme " par le test du $x^{2}$. 
TABLEAU I

Ségrégations observées pour les types pigmentaires: détails.

Segregations for pigmentation (détails)

\begin{tabular}{|c|c|c|c|c|c|c|c|c|c|c|c|}
\hline \multicolumn{2}{|r|}{ Croisement } & \multirow{3}{*}{$\begin{array}{c}\text { Père } \\
\text { No }\end{array}$} & \multirow{3}{*}{$\begin{array}{c}\text { Mère } \\
\text { No }\end{array}$} & \multicolumn{8}{|c|}{ Descendants } \\
\hline \multirow{2}{*}{ No } & \multirow{2}{*}{ Type } & & & \multicolumn{2}{|c|}{ Noir } & \multicolumn{2}{|c|}{ Domino } & \multicolumn{2}{|c|}{ Gris } & \multicolumn{2}{|c|}{ Chabin } \\
\hline & & & & M & $\mathrm{F}$ & $\mathrm{M}$ & $\mathbf{F}$ & $\mathbf{M}$ & $\mathbf{F}$ & $\mathbf{M}$ & $\mathbf{F}$ \\
\hline $\mathbf{r}^{-}$ & domino $\times$domino.. & $\begin{array}{l}826 \\
826 \\
826\end{array}$ & $\begin{array}{r}307 \\
694 \\
743 \\
\end{array}$ & $\begin{array}{l}0 \\
0 \\
0 \\
\end{array}$ & $\begin{array}{l}0 \\
0 \\
0\end{array}$ & $\begin{array}{l}6 \\
6 \\
3 \\
\end{array}$ & $\begin{array}{l}6 \\
5 \\
7\end{array}$ & & & & \\
\hline & & & & o & 0 & I 5 & I 8 & o & o & 0 & o \\
\hline 2 & noir $\times$ noir. . . . & $\begin{array}{l}428 \\
428 \\
428 \\
428 \\
\text { GG } \\
428 \\
428 \\
428 \\
428 \\
\end{array}$ & $\begin{array}{r}\text { OI } \\
\text { O3 } \\
\text { I I } \\
2 \text { I } \\
24 \\
25 \\
34 \\
49 \\
447 \\
\end{array}$ & $\begin{array}{r}\text { I0 } \\
8 \\
5 \\
3 \\
5 \\
5 \\
7 \\
3 \\
4 \\
\end{array}$ & $\begin{array}{r}5 \\
5 \\
4 \\
3 \\
2 \\
6 \\
6 \\
\text { 10 } \\
2 \\
\end{array}$ & $\begin{array}{l}\text { I } \\
3 \\
\text { I } \\
\text { I } \\
2 \\
0 \\
3 \\
0 \\
\text { I }\end{array}$ & $\begin{array}{l}2 \\
2 \\
0 \\
0 \\
0 \\
0 \\
3 \\
0 \\
2 \\
\end{array}$ & & & & \\
\hline & & & & 50 & 43 & I 2 & 9 & o & o & $\mathbf{o}$ & o \\
\hline 3 & noir $\times$ domino.. & $\begin{array}{l}428 \\
428 \\
428 \\
428 \\
429 \\
\text { GG } \\
\text { GG } \\
\text { GG }\end{array}$ & $\begin{array}{r}08 \\
40 \\
43 \\
307 \\
33 \\
26 \\
\text { I } 16 \\
307 \\
\end{array}$ & $\begin{array}{l}0 \\
\text { I } \\
\text { I } \\
2 \\
0 \\
\mathrm{I} \\
5 \\
\mathrm{I}\end{array}$ & $\begin{array}{l}\mathbf{I} \\
3 \\
3 \\
\mathbf{1} \\
3 \\
3 \\
3 \\
2 \\
\end{array}$ & $\begin{array}{l}0 \\
0 \\
I \\
3 \\
I \\
I \\
4\end{array}$ & $\begin{array}{l}4 \\
2 \\
5 \\
1 \\
0 \\
3 \\
2 \\
\end{array}$ & & & & \\
\hline & & & & I I & 19 & IO & 17 & o & o & o & o \\
\hline 4 & domino $\times$ noir.. & $\begin{array}{l}826 \\
826 \\
826 \\
826 \\
826 \\
826 \\
826\end{array}$ & $\begin{array}{l}591 \\
601 \\
656 \\
693 \\
711 \\
716 \\
739 \\
\end{array}$ & $\begin{array}{l}5 \\
0 \\
2 \\
0 \\
0 \\
0 \\
2\end{array}$ & $\begin{array}{l}3 \\
I \\
4 \\
0 \\
0 \\
0 \\
2 \\
\end{array}$ & $\begin{array}{l}0 \\
5 \\
\text { I } \\
3 \\
4 \\
4 \\
3 \\
\end{array}$ & $\begin{array}{l}\text { O } \\
\text { I } \\
\text { o } \\
4 \\
4 \\
0 \\
2 \\
\end{array}$ & & & & \\
\hline & & & & 9 & Io & 20 & I I & o & o & o & o \\
\hline 5 & gris $\times$ noir $\cdot \cdot \cdot \cdot$ & $\begin{array}{l}33^{8} \\
33^{8} \\
33^{8} \\
33^{8} \\
33^{8} \\
33^{8} \\
33^{8} \\
33^{8}\end{array}$ & $\begin{array}{r}03 \\
04 \\
24 \\
49 \\
291 \\
292 \\
446 \\
447 \\
642 \\
\end{array}$ & $\begin{array}{l}0 \\
5 \\
0 \\
1 \\
1 \\
0 \\
0 \\
2 \\
2\end{array}$ & $\begin{array}{l}2 \\
5 \\
\mathrm{I} \\
3 \\
5 \\
\mathrm{I} \\
\mathrm{o} \\
\mathrm{r} \\
3 \\
\end{array}$ & $\begin{array}{l}0 \\
0 \\
I \\
0 \\
0 \\
0 \\
0 \\
2 \\
\text { I }\end{array}$ & $\begin{array}{l}\mathbf{I} \\
0 \\
0 \\
0 \\
0 \\
0 \\
0 \\
\mathrm{I} \\
3 \\
\end{array}$ & $\begin{array}{l}\text { I } \\
3 \\
3 \\
2 \\
2 \\
0 \\
2 \\
0 \\
0\end{array}$ & $\begin{array}{l}\text { I } \\
3 \\
3 \\
2 \\
2 \\
2 \\
\text { I } \\
0 \\
\text { I }\end{array}$ & $\begin{array}{l}\text { I } \\
0 \\
3 \\
0 \\
0 \\
0 \\
0 \\
0 \\
0\end{array}$ & $\begin{array}{l}\mathbf{1} \\
0 \\
0 \\
0 \\
0 \\
0 \\
0 \\
0 \\
0\end{array}$ \\
\hline & & & & I I & $2 \mathrm{I}$ & 4 & 5 & 13 & I 5 & 4 & $\mathbf{I}$ \\
\hline 6 & gris $\times$ domino . . . & $33^{8}$ & $\begin{array}{r}40 \\
116\end{array}$ & $\begin{array}{l}3 \\
3\end{array}$ & $\begin{array}{l}2 \\
2\end{array}$ & $\begin{array}{l}2 \\
4\end{array}$ & $\begin{array}{l}3 \\
4\end{array}$ & $\begin{array}{l}\text { I } \\
\text { I }\end{array}$ & $\begin{array}{l}0 \\
0\end{array}$ & $\begin{array}{l}0 \\
0\end{array}$ & $\begin{array}{l}0 \\
0\end{array}$ \\
\hline 7 & domino $\times$ gris... & 826 & 729 & $\mathbf{I}$ & 2 & & & 4 & $\mathbf{I}$ & 0 & 0 \\
\hline & & & & 7 & 6 & 6 & 7 & 6 & $\mathbf{I}$ & 0 & o \\
\hline
\end{tabular}




\section{Résultats}

Les résultats détaillés sont donnés dans le tableau I où on a opéré certains regroupements pour les truies ayant eu plusieurs portées avec le même verrat d'où la non correspondance avec le nombre de portées (48) annoncé plus haut.

\section{Discussion}

\section{I. - Les croisements entre noirs et domino}

Ces croisements ne produisent que des noirs et des dominos. Ils s'interprètent assez simplement en supposant un diallélisme autosomal, le noir étant un simple dominant sur le domino. Dans le tableau 2 on a testé cette hypothèse en admettant en outre que tout les noirs sont hétérozygotes, ce qui est évident déjà pour les mâles à l'examen du tableau $I$.

TABLEAU 2

Analyse des croisements entre les phénotypes noir et domino

Analyses of crossings betwen black and domino phenotypes

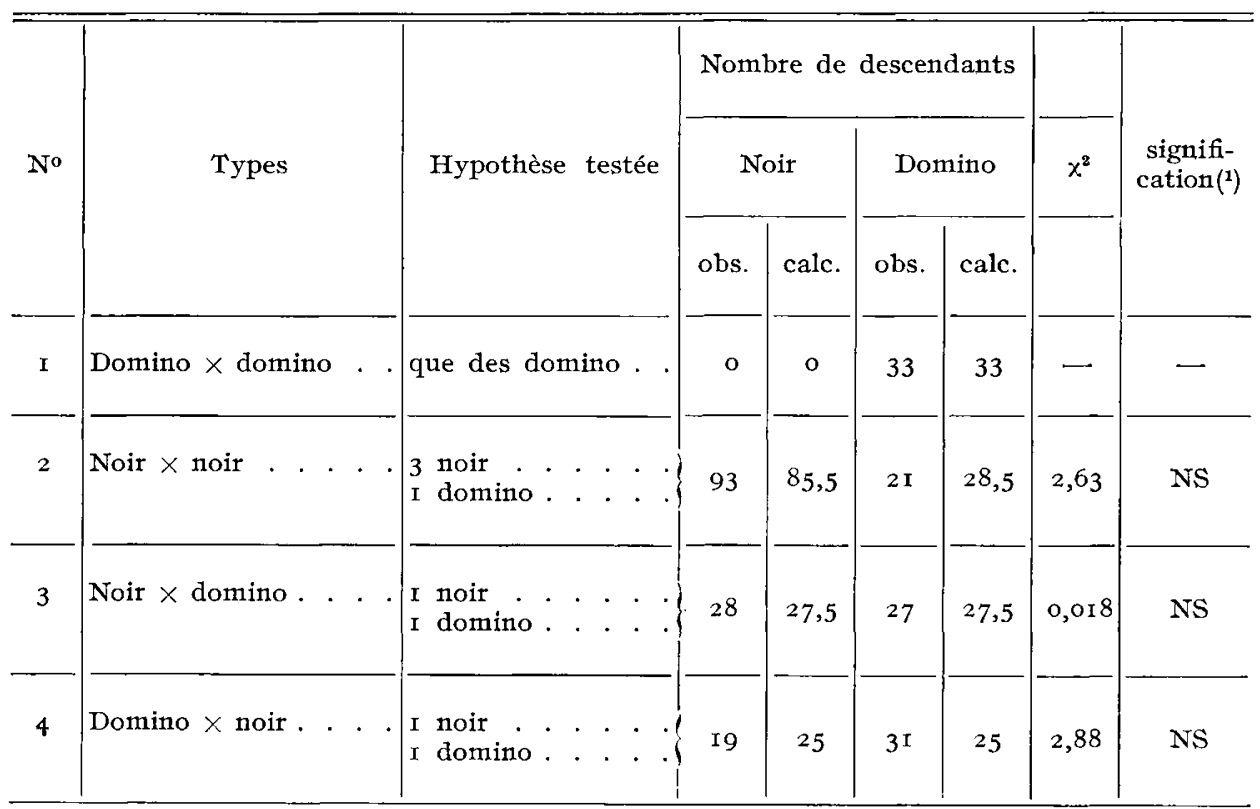

(1) N.S. non significatif au seuil 0,05 . 
I1 s'agit ensuite de préciser auquel des deux principaux loci de coloration (A Agouti ou E Extension, ProTA et SEARLE, I978) se fait la ségrégation. En effet si, pour des raisons d'homologie, il y a de fortes chances pour que le phénotype

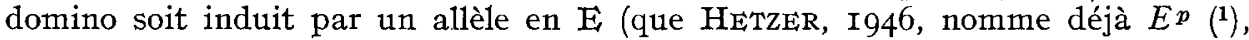
cf. aussi OLLIVIER et SELLIER, I980 pour revue) qui serait l'équivalent de $e^{f}$, bringeure japonnaise du lapin ou de epécaille de tortue du cobaye (SEARLE, I968), la ségrégation observée peut aussi bien résulter d'une relation de dominance en $\mathrm{E}$ que d'une alternative allélique en $\mathrm{A}$ avec action épistatique d'un allèle de $\mathrm{A}$ sur $\mathrm{E}$.

L'homologie des loci de coloration chez les mammifères que l'on doit encore invoquer ici nous indique que l'épistasie de $\mathrm{A}$ sur $\mathrm{E}$ est rarissime, donc peu vraisemblable. On est donc sans doute en présence d'une ségrégation en $\mathrm{E}$ de $E^{p}$ avec un autre allèle qui peut être $E$, extension normale du noir ou $E^{d}$, noir dominant $\left({ }^{2}\right)$.

Il est difficile de trancher entre ces deux possibilités sans connaître la formule au locus Agonti, la même pour tous les animaux puisque, apparemment, ce locus est homozygote dans le cas qui nous intéresse. Si 1'allèle en Agouti est a (noir recessif) on peut avoir soit $E$ soit $E^{d}$. Si c'est un autre allèle que $a$ alors l'éventualité $E$ sera sans doute exclue car la formule $E E A^{x} A^{x}$ ne donne du noir uniforme que si $\mathrm{A}^{x}$ est $a$.

Il est difficile de conclure avec certitude car on connaît mal chez le Porc les interactions entre allèles en A et en E. En particulier il se pourrait que le phénotype domino (tâches toutes noires sur fond tout rouge, sans poils zonés) ne s'observe qu'en présence de $a a$ comme on le pensait déjà à propos du phénotype coloré domino du Piétrain (Lauvergne et Oldivier, rg66). D'ailleurs, Searle (r968) nous rappelle que $a$ est l'allèle en $A$ gouti le plus fréquent chez le porc. Cela renforcerait 1'hypothèse selon laquel l'allèle en ségrégation avec $E^{p}$ serait $E$ ce que nous admettrons dans la suite de cet article.

\section{2. - Les croisements oì intervient le gris}

L'hypothèse la plus simple pour interpréter les croisements 5, 6 et 7 des tableaux I et 2 est de supposer qu'à un loci indépendant de $\mathrm{E}$ existe une allèle dominant qui, sur fond noir, donne du gris et sur fond domino donne le blanc sale du chabin. C'est cette hypothèse qui est testée dans le tableau 3, en supposant que les reprođucteurs noirs aussi bien que les gris sont hétérozygotes $E^{p} E$ comme on vient de le démontrer et que les gris sont en outre hétérozygotes pour un gène de grisonnement situé à un autre locus, indépendant de $\mathrm{E}$.

On voit que si cette hypothèse est acceptable pour le croisement $n^{0} 5$ par contre pour les croisés issus des types domino $\times$ gris et réciproque on observe un déficit d'animaux chabin d'ailleurs déjà sensible dans le croisement précédent mais moins facilement décelable, vu la faible proportion attendue.

L'interprétation la plus simple est que l'effet " chabin " de l'hétérozygote gris sur fond domino $\left(E^{p} E^{p}\right)$ est à pénétrance incomplète, comme le prouverait également l'excès de domino observé dans les croisements 6 et 7 .

Cette variation grise (roan en anglais) est connue des anciens auteurs qui $1^{\prime}$ 'ont attribué à l'effet d'un gène $I^{d}$ (HETZER I948). Le gène $I^{d}$ allèle du blanc domi-

(1) Que SEARLE (r968) nomme red and black rouge et noir ou tigering (tigré) lut attribuant le symbole $e^{j}$.

(2) En E toujours selon SEARLE (I968) on aurait les rapports de dominance suivants $E^{d}>E^{2}>E^{p}$ (ou $\left.e^{j}\right)>e$ extension récessive du rouge. 


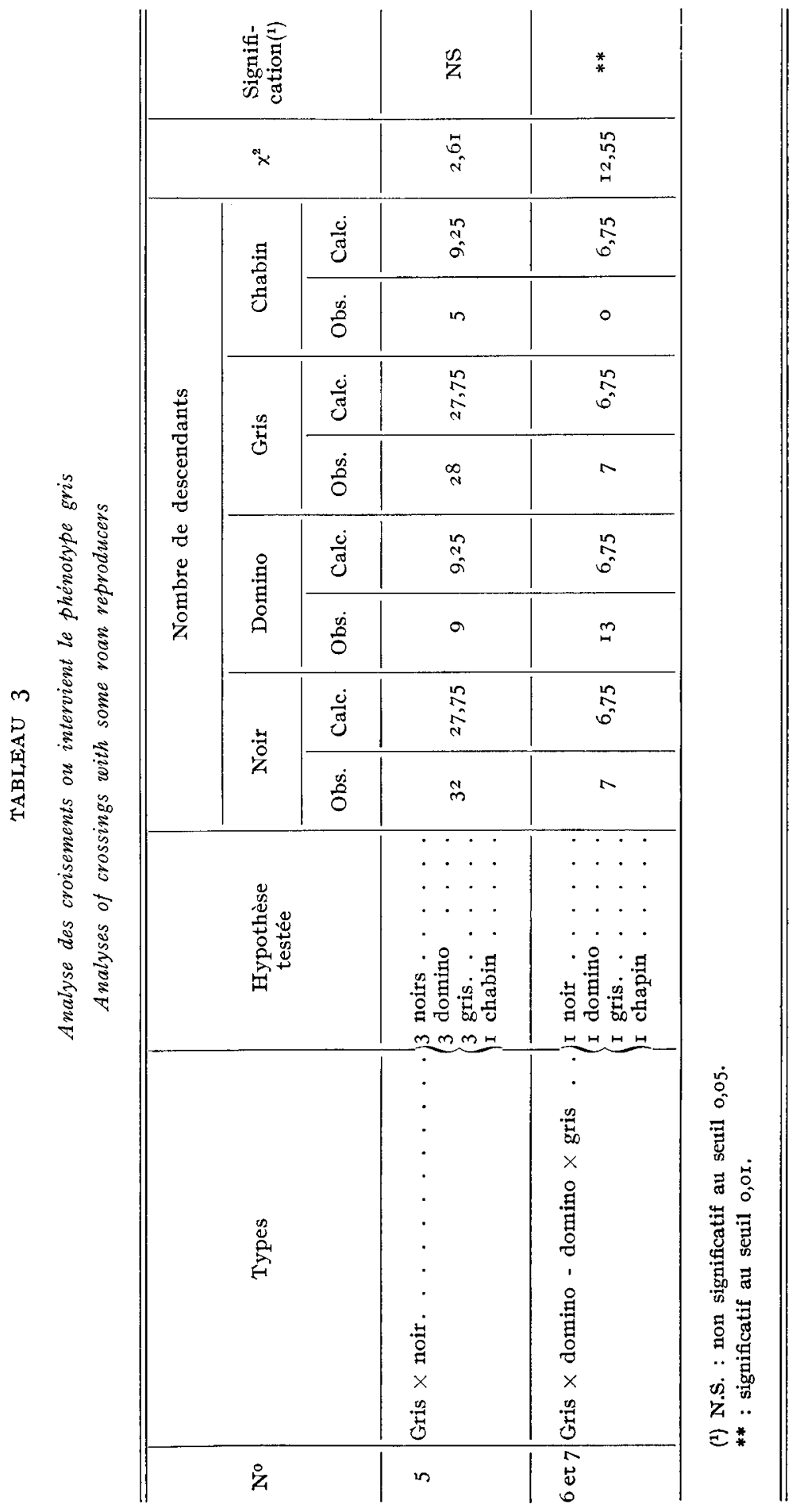


nant $(I)$ du Large white lui serait recessif tout en étant dominant sur l'allèle normal $i: I>I^{d}>i$. Il est intéressant de noter que cette allèle pourrait, dans certains cas au moins, manifester une pénétrance incomplète.

\section{3. - L'hétérozygotie $E^{p} E$ des reproducteurs noirs et gris}

On a du mal à expliquer l'hétérozygotie rencontrée chez pratiquement tous les mâles noirs ou gris, comme on peut voir dans le tableau $\mathrm{I}$, où certains sont testés pour ce caractère et chez la plupart des femelles comme le montrent les rapports de ségrégation. S'agit-il d'une caractéristique de la population indigène de l'île ou le gène $E$ serait peu fréquent oì alors simplement d'une dérive génétique introduite lors de la constitution du troupeau expérimental de Petit-Bourg, à partir de peu d'individus?

Quant à la possible influence d'une sélection en faveur de reproducteurs hétérozygotes, d'après ce que nous savons, si elle a existé, elle s'est effectuée inconsciemment.

\section{Conclusion}

Au terme de notre étude sur les variants colorés (pigmentaires) du porc Créole de la Guadeloupe, il semble que l'on puisse distinguer la présence de 3 allèles mutés déjà connus, au moins dans les races européennes :

- $a$ au locus A (noir recessif),

- $E^{p}$ au locus $\mathrm{E}$ (noir et rouge ou tigré),

- Id au locus I (gris ou rouan).

Cela semble peu au regard des 14 variants déjà détectés chez le porc (OLLIVIER et SELLIER, I980) mais il est à noter que les variations de panachure avec au moins, un allèle muté n'ont pas été étudiées et que l'on est parti d'une population de reproducteurs limitée (seulement 6 mâles au total) qui au départ n'avait pas était constituée dans le but de rassembler tous les variants colorés existant dans 1'île.

$$
\text { Rę̧u pour publication en février } 1980 .
$$

\section{Summary}

\section{Study of some variants in the Criollo pig of Guadeloupe}

In the pig breeding unit of C.R.A.A.G. at Petit-Bourg, Guadeloupe (French West Indies) mutants at two already apparently known and non linked loci have been demonstrated : $E$ normal extension of black dominant on $E^{p}$ black and red or tigering (sometimes quoted $e^{j}$ ) at the Extension locus (E) and $I^{d}$ (roan) dominant on $i$, intense, at the Roan (I) locus.

Apparently there is no segregation at the Agouti locus (A) which could be homozygous aa (recessive black) for all the animals of the experience.

Some animals were piebald (with white belt or socks) but, without precise sketches of the white patterns, the genetic study could not be carried out. 
The coloured genetic formula of the black pigs could be $E E$ (or $E E^{p}$ ) $a a$ ii, that of the tigering animal locally called "domino " and quite variable in the black and red pattern as well as in the colour of red background being $E^{p} E^{p}$ aa $i i$.

The grey roan pigs could be $E E$ (or $E E^{p}$ ) a a Id $i$ and the socalled "chabin" (dirty white animals) $E^{p} E^{p}$ aa $I^{d} i$. The penetrance of " chabin " phenotype among animals with this last formula does not seem to be complete.

\section{Références bibliographiques}

DUtertre J. B., I667-1671. Histoire générale des Antilles, 3 vol. 4 tomes, Nouvelle réédition I958, Société d'Histoire de la Martinique C.E.P. Fort de France.

HETZER H. O., I946. Inheritance of coat color in Swine. V. Results of Landrace by DurocJersey crosses. J. Heved., 37, 21 7-224.

HETZER H. O., I948. Inheritance of coat color in Swine. VII. Results of Landrace by Hampshive crosses. J. Heved., 39, I23-1 28.

I.ABAT R. P., I 722. Nouveau voyage aux Isles de l'Amérique. Cavelier, Paris, 6 vol. (nombreuses rééditions).

LAUVERGNe J. J., OLLIVIER I., r966. A propos de colorations observées lors de croisements entre porcs de Piétrain et porcs Large White. Ann. Génét., 9, 39-41.

LE MENTEC J. C., I970. Étude monographique du porc local en Guadeloupe. Bull. tech. Inf. Min. Agric. (25I), 435-446.

OlLiVIER L., SELLIER P., I980. Pig, in Robinson R. Handbook of Mammalian Genetics. Garland, New-York (sous presse).

Prota G., Searte A. G., 1978. Biochemical sites of genes action for melanogenesis in mammals. Ann. Génét. Sél. anim., 10, I-8.

SEARIE A. G., I968. SEARLE A. G., 1968. Comparative Genetics of Coat Colour in Mammals. Logos, Academic Press, London, New York, I 23, I 24, I29, I96-199. 